\title{
SEPARATION AXIOMS FOR INTERVAL TOPOLOGIES
}

\author{
MARCEL ERNE
}

\begin{abstract}
In Theorem 1 of this note, results of Kogan [2], Kolibiar [3], Matsushima [4] and Wolk [7] concerning interval topologies are presented under a common point of view, and further characterizations of the $T_{2}$ axiom are obtained. A sufficient order-theoretical condition for regularity of interval topologies is established in Theorem 2 . In lattices, this condition turns out to be equivalent both to the $T_{2}$ and to the $T_{3}$ axiom. Hence, a Hausdorff interval topology of a lattice is already regular. However, an example of a poset is given where the interval topology is $T_{2}$ but not $T_{3}$.
\end{abstract}

1. Introduction. It is well known that the interval topology of any chain satisfies each of the separation axioms $T_{0}, \ldots, T_{5}$ (cf. [6, p. 67]). This no longer holds if chains are replaced with arbitrary lattices or posets. Although the interval topology of any poset is $T_{1}$, there are even complete lattices for which the interval topology is not $T_{2}$. A necessary and sufficient condition for the interval topology to be $T_{2}$ has been given by M. Kolibiar [3]. In this note, we mainly study under which conditions the interval topology may be regular. Specifically, in the case of lattices we shall find that for interval topologies, the $T_{2}$ axiom and the $T_{3}$ axiom are equivalent.

2. Basic notations. Let $X$ be an arbitrary poset, the partial ordering of which is indicated by the symbol $\leqslant$. For $Y \subset X, Y^{\dagger}$ and $Y^{*}$ denote the set of all lower and upper bounds of $Y$, respectively. The sets

$$
x^{\dagger}:=\{x\}^{\dagger}=\{y \in X: y<x\} \text { and } x^{*}:=\{x\}^{*}=\{y \in X: x<y\}
$$

are referred to as closed rays. Every closed ray, every set of the form

$$
[x, z]=x^{*} \cap z^{\dagger}=\{y \in X: x<y<z\},
$$

and the entire set $X$ are called (closed) intervals. The interval topology $\mathfrak{T}$ on $X$ is the smallest topology in which all intervals are closed sets. Thus the interval topology (considered as the collection of all open sets) is generated by the set-complements of all closed rays. In particular, each singleton

$$
\{x\}=[x, x]
$$

is closed in $\mathfrak{T}$, and one has

LEMMA 1. The interval topology of any poset is $\mathbf{T}_{1}$.

Received by the editors December 11, 1978 and, in revised form, April 6, 1979 and July 20, 1979. AMS (MOS) subject classifications (1970). Primary 06A45, 54F05.

Key words and phrases. Interval topology, separation axioms, regular, normal, finitely separable. 
3. The $\mathrm{T}_{2}$ axiom. For a subset $W$, let $N(W)$ denote the set of all elements in $X$ which are neither upper nor lower bounds of $W$, i.e.

$$
N(W):=X \backslash\left(W^{\dagger} \cup W^{*}\right) .
$$

We shall write $N(x)$ for $N(\{x\})$ and $N(x, y)$ for $N(\{x, y\})$. Thus $N(x)$ consists of all elements not comparable with $x$. A set $Y \subset X$ is finitely separable if there is a finite subset $V$ of $Y$ such that every element of $Y$ is comparable with some element of $V$. For the sake of brevity, we put

$$
\begin{aligned}
& \downarrow Y:=\{x \in X: x \leqslant y \text { for some } y \in Y\}=\bigcup\left\{y^{\dagger}: y \in Y\right\}, \\
& \uparrow Y:=\{x \in X: y \leqslant x \text { for some } y \in Y\}=\bigcup\left\{y^{*}: y \in Y\right\} .
\end{aligned}
$$

Hence a set $Y \subset X$ is finitely separable iff $Y \subset \downarrow V \cup \uparrow V$ for some finite $V \subset Y$. The following assertion is clear by the definition of the interval topology.

LEMMA 2. The sets $X \backslash(\downarrow Y \cup \uparrow Z)$, where $Y$ and $Z$ run over all finite subsets of $X$, form a base of the interval topology.

As an easy consequence, one obtains

LEMMA 3. The interval topology $\mathfrak{I}$ of any poset $X$ is $\mathrm{T}_{2}$ iff for all $x \neq y$, there are finite sets $Y, Z$ with $Y \cap\{x, y\}^{*}=\varnothing, Z \cap\{x, y\}^{\dagger}=\varnothing, X=\downarrow Y \cup \uparrow Z$.

Proof. If $\mathfrak{T}$ is $\mathrm{T}_{2}$ then for $x \neq y$, we can find finite sets $Y_{x}, Z_{x}, Y_{y}, Z_{y}$ such that $x \in U:=X \backslash\left(\downarrow Y_{x} \cup \uparrow Z_{x}\right), \quad y \in V:=X \backslash\left(\downarrow Y_{y} \cup \uparrow Z_{y}\right), \quad U \cap V=\varnothing$. Thus $Y:=Y_{x} \cup Y_{y}$ and $Z:=Z_{x} \cup Z_{y}$ have the required properties.

Conversely, let $Y, Z$ be finite sets such that $Y \cap\{x, y\}^{*}=\varnothing, Z \cap\{x, y\}^{\dagger}=\varnothing$, $X=\downarrow Y \cup \uparrow Z$. Then $U:=X \backslash\left(\downarrow\left(Y \backslash x^{*}\right) \cup \uparrow\left(Z \backslash x^{\dagger}\right)\right)$ and $V:=X \backslash\left(\downarrow\left(Y \backslash y^{*}\right)\right.$ $\cup \uparrow\left(Z \backslash y^{\dagger}\right)$ ) are disjoint open neighbourhoods of $x$ and $y$, respectively.

LEMMA 4. For any subset $W$ of $X$ containing at least two elements, $N(W)$ is finitely separable iff there are finite sets $Y, Z$ with $Y \cap W^{*}=\varnothing, Z \cap W^{\dagger}=\varnothing, \downarrow Y \cup \uparrow Z$ $=X$.

Proof. Suppose $V \subset N(W) \subset \downarrow V \cup \uparrow V,|V|<\infty$. Since $|W|>2$, we can choose $y, z \in W$ with $z \nless y$. The sets $Y:=V \cup\{y\}$ and $Z:=V \cup\{z\}$ have the required properties. Conversely, assume $Y \cap W^{*}=\varnothing, Z \cap W^{\dagger}=\varnothing, \downarrow Y \cup \uparrow Z=$ $X$ for some finite sets $Y, Z$. Then $V:=N(W) \cap(Y \cup Z)$ is a finite subset of $N(W)$, and it is easily verified that $N(W) \subset \downarrow V \cup \uparrow V$.

COROllary 1. If $N(W)$ is finitely separable and $|W|>2$ then for all sets $Y$ containing $W, N(Y)$ is also finitely separable.

Proof. $W \subset Y$ implies $Y^{\dagger} \subset W^{\dagger}$ and $Y^{*} \subset W^{*}$, so we can apply Lemma 4.

By an antichain, we mean a set of pairwise incomparable elements. Adjoining a least element 0 and a greatest element 1 to an infinite antichain, a complete lattice is obtained in which $N(0)$ and $N(1)$ are finitely separable (being empty) while $N(0,1)$ is not. 
THEOREM 1. Consider the following statements for a partially ordered set $X$ :

(a) $X$ contains no infinite antichain.

(a') Every subset of $X$ is finitely separable.

(b) For all $x \in X$, the set $N(x)$ is finitely separable.

(b') For every subset $W$ of $X, N(W)$ is finitely separable.

(c) For all $x \neq y$, the set $N(x, y)$ is finitely separable.

(c') For every subset $W$ of $X$ containing more than one element, $N(W)$ is finitely separable.

(c") The interval topology of $X$ is $\mathrm{T}_{2}$.

One has the following implications:

$$
(a) \Leftrightarrow\left(a^{\prime}\right) \Rightarrow(b) \Leftrightarrow\left(b^{\prime}\right) \Rightarrow(c) \Leftrightarrow\left(c^{\prime}\right) \Leftrightarrow\left(c^{\prime \prime}\right) \text {. }
$$

Proof. The equivalence of (a) and $\left(a^{\prime}\right)$ is clear. In view of Lemma 3, Lemma 4, and Corollary 1, it only remains to show the implication (b) $\Rightarrow$ (c): Assume $x<z<y$ or $y<z<x$ for some $z$. Then we find a finite set $V \subset N(z)$ with $N(z) \subset \downarrow V \cup \uparrow V$, and $W:=V \cup\{z\}$ is a finite subset of $N(x, y)$ with $\downarrow W \cup \uparrow W$ $=X$. In particular, $N(x, y)$ is finitely separable. In all other cases, we obtain $N(x, y)=N(x) \cup N(y)$, and then hypothesis (b) ensures again that $N(x, y)$ is finitely separable.

The implication $(a) \Rightarrow\left(c^{\prime \prime}\right)$ is due to E. S. Wolk [7], and $(b) \Rightarrow\left(c^{\prime \prime}\right)$ has been shown by $Y$. Matsushima [4] who also disproved the implication (b) $\Rightarrow$ (a) by a counterexample. The equivalence of $(c)$ and $\left(c^{\prime \prime}\right)$ has been stated without proof by M. Kolibiar in [3]. Moreover, Kolibiar has constructed an example where ( $\left.c^{\prime \prime}\right)$ but not (b) holds. Finally, as F. S. Northam [5] has remarked, (c") implies that every open interval $] x, y[=\{z: x<z<y\}$ be finitely separable.

4. The $T_{3}$ axiom. The investigation of regularity for interval topologies involves some more effort. First of all, we reduce the separation of points from closed sets to subbasic closed sets.

LEMMA 5. The interval topology $\mathfrak{Z}$ of a poset $X$ is regular (i.e. $\left.\mathrm{T}_{3}\right)$ iff the following condition and its dual hold:

(*) For all $x, y \in X$ with $x \nless y$, there exist disjoint $\mathfrak{Z}$-open sets $U, V$ with $x \in U$, $y^{\dagger} \subset V$.

Proof. The necessity of this condition is clear. To show sufficiency, let $A$ be an arbitrary $\mathfrak{Z}$-closed set with $x \notin A$. Then $A$ can be written as an intersection of sets $A_{i}$ each of which is a finite union of closed rays, say

$$
A_{i}=\downarrow Y_{i} \cup \uparrow Z_{i}
$$

where $Y_{i}$ and $Z_{i}$ are finite subsets of $X(i \in I) . x \notin A$ implies $x \notin A_{i}$ for some $i \in I$, and it follows $x \nless y$ for all $y \in Y_{i}, z \nless x$ for all $z \in Z_{i}$. Hence we find open sets $U_{y}, V_{y}$ with $x \in U_{y}, y^{\dagger} \subset V_{y}, U_{y} \cap V_{y}=\varnothing\left(y \in Y_{i}\right)$, and open sets $U_{z}^{\prime}$, $V_{z}^{\prime}$ with $x \in U_{z}^{\prime}, z^{*} \subset V_{z}^{\prime}, U_{z}^{\prime} \cap V_{z}^{\prime}=\varnothing\left(z \in Z_{i}\right) . \quad U:=\cap\left\{U_{y}: y \in Y_{i}\right\} \cap$ $\cap\left\{U_{z}^{\prime}: z \in Z_{i}\right\}$ is a neighbourhood of $x$, and $V:=\cup\left\{V_{y}: y \in Y_{i}\right\} \cup$ $\cup\left\{V_{z}^{\prime}: z \in Z_{i}\right\}$ is an open set disjoint from $U$ and containing $A_{i}$. In particular, $A \subset V$, and we have separated $x$ and $A$ by disjoint open sets $U, V$. 
Remember that the interval topology is $T_{2}$ iff the sets

$$
N(x, y)=X \backslash\left(\left(x^{\dagger} \cap y^{\dagger}\right) \cup\left(x^{*} \cap y^{*}\right)\right) \quad(x \neq y)
$$

are finitely separable. Now defining

$$
\begin{aligned}
M(x, y) & :=X \backslash\left(\left(x^{\dagger} \cap y^{\dagger}\right) \cup x^{*}\right), \\
P(x, y) & :=X \backslash\left(\left(x^{*} \cap y^{*}\right) \cup y^{\dagger}\right),
\end{aligned}
$$

we can show a similar result concerning the $T_{3}$ axiom:

THEOREM 2. For the interval topology of a poset $X$ to be regular it is sufficient that for all $x, y \in X$ with $x \nless y$, the sets $M(x, y)$ and $P(x, y)$ are finitely separable. In lattices, this condition is also necessary.

Proof. Suppose $x \nless y$. We have to separate $x$ and $y^{\dagger}$ by open sets. (Dual arguments show that $y$ and $x^{*}$ can be separated.) By hypothesis, there is a finite set $W$ with $W \subset M(x, y) \subset \downarrow W \cup \uparrow W$. Define

$$
Y:=W \cup\{y\}, \quad Z:=W \backslash x^{\dagger}, \quad Z^{\prime}:=\left(W \backslash y^{\dagger}\right) \cup\{x\} .
$$

Then $Y, Z, Z^{\prime}$ are finite sets such that

(1) $W \subset Y$,

(2) $W \cap x^{\dagger} \cap y^{\dagger}=\varnothing, W=\left(W \backslash x^{\dagger}\right) \cup\left(W \backslash y^{\dagger}\right) \subset Z \cup Z$;

(3) $x \notin \downarrow Y$ (since $x \nless y$ and $W \cap x^{*}=\varnothing$ ),

(4) $x \notin \uparrow Z$ (since $Z \cap x^{\dagger}=\varnothing$ ),

(5) $y^{\dagger} \cap \uparrow Z^{\prime}=\varnothing\left(\right.$ since $\left.y^{\dagger} \cap Z^{\prime}=\varnothing\right)$,

(6) $X \backslash M(x, y)=\left(x^{\dagger} \cap y^{\dagger}\right) \cup x^{*} \subset \downarrow Y \cup \uparrow Z$;

(7) $M(x, y) \subset \downarrow W \cup \uparrow W \subset \downarrow Y \cup \uparrow Z \cup \uparrow Z^{\prime}$ (by 1 and 2).

Hence,

$$
\begin{aligned}
x \in U & :=X \backslash(\downarrow Y \cup \uparrow Z) \in \mathfrak{T} \quad \text { (by } 3 \text { and 4), } \\
y^{\dagger} \subset V & :=X \backslash \uparrow Z^{\prime} \in \mathfrak{T} \quad \text { (by 5), } \\
U \cap V & =\varnothing \quad \text { (by } 6 \text { and 7). }
\end{aligned}
$$

In a lattice with join $\vee$ and meet $\wedge$, one has

$$
\begin{aligned}
& N(x, y)=X \backslash\left((x \wedge y)^{\dagger} \cup(x \vee y)^{*}\right), \\
& M(x, y)=X \backslash\left((x \wedge y)^{\dagger} \cup x^{*}\right), \\
& P(x, y)=X \backslash\left(y^{\dagger} \cup(x \vee y)^{*}\right) .
\end{aligned}
$$

Furthermore, $x \neq y$ implies $x \wedge y<x \vee y$, and $x \nless y$ implies $x \wedge y<x$ and $y<x \vee y$. Accordingly, the second statement in Theorem 2 can be sharpened to

THEOREM 3. In a lattice, the following statements are equivalent:

(a) For all $x, y$ with $y<x$, the set $X \backslash\left(y^{\dagger} \cup x^{*}\right)$ is finitely separable.

(b) The interval topology is $\mathrm{T}_{2}$.

(c) The interval topology is $\mathrm{T}_{3}$ (regular).

The equivalence of (a) and (b) is due to S. A. Kogan [2].

In lattices, Matsushima's condition (Theorem 1, b) implies that for all $x, y$ with $x \nless y, M(x, y)$ and $P(x, y)$ are finitely separable. This implication no longer holds 
in arbitrary posets, and not even in semilattices.

EXAMPLE 1. Adjoin three new elements $x, y, z$ to the set $\mathbf{Z}$ of all integers and define a partial ordering on $X:=\mathbf{Z} \cup\{x, y, z\}$ by setting

$$
\begin{aligned}
& x^{\dagger}:=\mathbf{Z} \cup\{x\}, \quad y^{\dagger}:=\{a \in \mathbf{Z}: a>0\} \cup\{y\}, \quad z^{\dagger}:=X, \\
& a^{\dagger}:=\{b \in \mathbf{Z}: 0<b<a\} \quad(a \in \mathbf{Z}, a>0), \\
& b^{\dagger}:=\{a \in \mathbf{Z}: 0<a<-b\} \cup\{b\} \quad(b \in \mathbf{Z}, b<0) .
\end{aligned}
$$

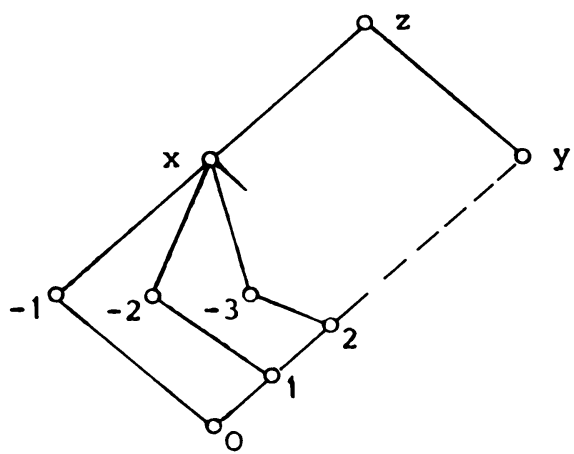

Then $X$ becomes a join-semilattice in which all points except $x$ are isolated in the interval topology. In particular, the separation axioms are trivially fulfilled. Moreover, an easy verification shows that for all $w \in X, N(w)$ is finitely separable. However, $M(x, y)$ is an infinite antichain and therefore not finitely separable. This example also shows that in general, the condition stated in Theorem 2 is not necessary for regularity.

Finally, let us construct a counterexample disproving the conjecture that Matsushima's condition might imply regularity in general. Thereby, we shall see that in arbitrary posets, the $T_{3}$ axiom is strictly stonger than the $T_{2}$ axiom.

EXAmple 2. For any integer $j>1$, let $p(j)$ denote the least prime divisor of $j$. Consider a set $X$ constituted by three sequences $\left(a_{n}\right),\left(b_{n}\right),\left(c_{n}\right)$, and define a partial ordering on $X$ by setting

$$
\begin{aligned}
& b_{j}<a_{i} \Leftrightarrow i \neq j \text { and }(i=1 \text { or } j=1 \text { or } p(j)<i), \\
& c_{k}<b_{j} \Leftrightarrow k \neq 1 \text { and } j \neq 1 \text { and } p(j)>k, \\
& c_{k}<a_{i} \Leftrightarrow k \neq 1 \text { and }(i=1 \text { or } k<i), \\
& c_{k}<c_{n} \Leftrightarrow n=1 \text { or } 1<k<n,
\end{aligned}
$$

while all other pairs of distinct elements are assumed to be incomparable. A straightforward computation shows that for all $w \in X$, the set $N(w)$ is finitely separable, and in particular, the interval topology is $T_{2}$. However, for $x:=a_{1}$ and $y:=c_{1}$, we shall see that $x$ and the closed ray $y^{\dagger}$ cannot be separated by open sets, disproving regularity. In fact, a neighbourhood base for the point $x$ is made up by the sets

$$
U_{i m}:=\left\{b_{j}: j>m, p(j)>i\right\} \cup\{x\} \quad(m>i),
$$


and for each prime number $p=k+1>2$, a neighbourhood base of $c_{k}$ is constituted by the sets

$$
V_{k n}=\left\{b_{j}: j>n, p(j)=p\right\} \cup\left\{c_{k}\right\} \quad(n>p) .
$$

All other points $c_{k}$ are isolated in the interval topology. Assume there would exist disjoint open sets $U, V$ such that $x \in U, y^{\dagger} \subset V$. Then we find some $m>i$ with $x \in U_{i m} \subset U$. Choose a prime number $p=k+1>m$. Then $c_{k} \in y^{\dagger} \subset V$ implies $c_{k} \in V_{k n} \subset V$ for some $n \geqslant p$. But for $j:=p^{n}$, we obtain $b_{j} \in U_{i m} \cap V_{k n}=\varnothing$, a contradiction.

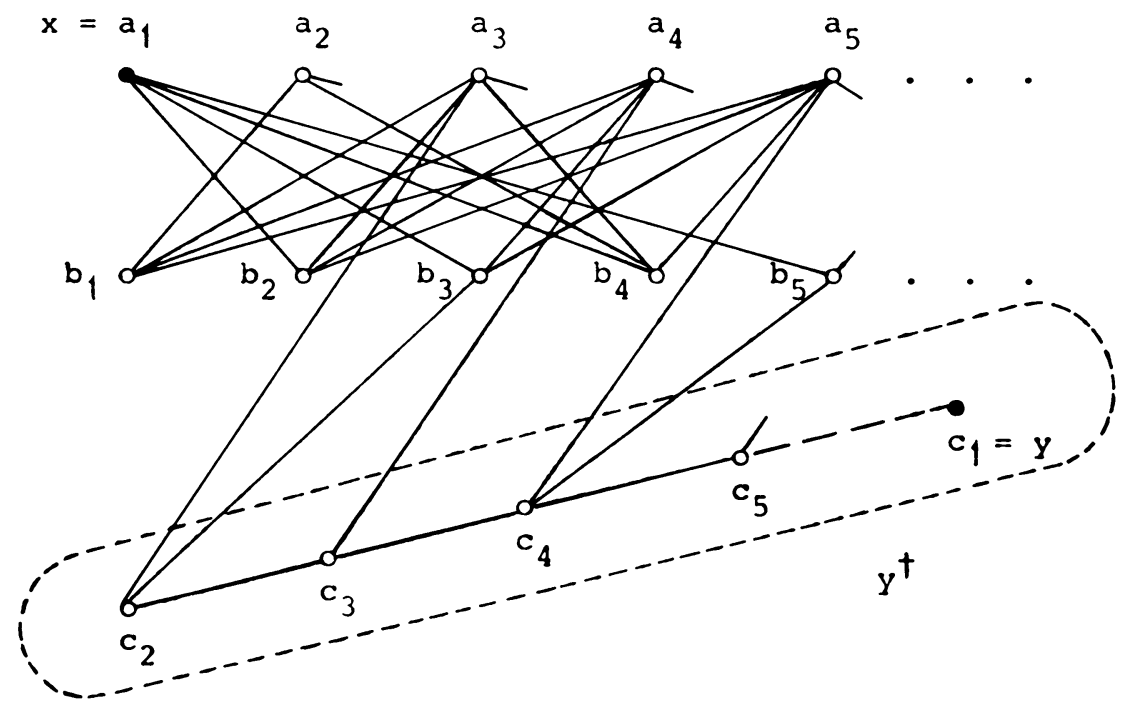

Concluding Remark. By a theorem of O. Frink [1], the interval topology of a lattice is compact iff the lattice is complete. Observing that a compact $T_{2}$ space is always normal (cf. [6, p. 25]), we infer that in complete lattices, the separation axioms $T_{2}, T_{3}$ and $T_{4}$ are trivially equivalent for the interval topology. It remains open whether this is true in arbitrary lattices.

\section{REFERENCES}

1. O. Frink, Topology in lattices, Trans. Amer. Math. Soc. 51 (1942), 569-582.

2. S. A. Kogan, Solution of three problems in lattice theory, Uspehi Mat. Nauk 11 (1956), 185-190.

3. M. Kolibiar, Bemerkungen über Intervalltopologie in halbgeordneten Mengen, General Topology and its Relations to Modern Analysis and Algebra (Proc. Sympos., Prague, 1961), Academic Press, New York; Publ. House Czech. Acad. Sci., Prague, 1962, pp. 252-253.

4. Y. Matsushima, Hausdorff interval topology on a partially ordered set, Proc. Amer. Math. Soc. 11 (1960), 233-235.

5. F. S. Northam, The interval topology of a lattice, Proc. Amer. Math. Soc. 4 (1953), 824-827.

6. L. A. Steen and J. A. Seebach, Jr., Counterexamples in topology, Holt, Rinchart and Winston, New York, 1970.

7. E. S. Wolk, Topologies on a partially ordered set, Proc. Amer. Math. Soc. 9 (1958), 524-529.

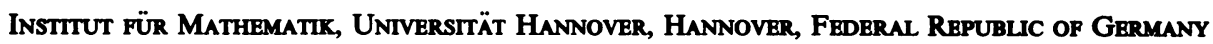

